\title{
Proton Therapy for Head and Neck Adenoid Cystic Carcinoma: Initial Clinical Outcomes
}

\author{
Okechukwu R. Linton, M.D.,* Michael G. Moore, M.D., ${ }^{\dagger}$ Joseph S. Brigance, M.D., ${ }^{\dagger}$ \\ Don-John Summerlin, D.M.D., M.S. ${ }^{\ddagger}$ Mark W. McDonald, M.D.,*§ \\ *Department of Radiation Oncology, ${ }^{\dagger}$ Department of Otolaryngology/Head \& Neck Surgery, \\ and ${ }^{\ddagger}$ Department of Pathology, \\ Indiana University School of Medicine, Indianapolis, Indiana, \\ §Indiana University Health Proton Therapy Center, Bloomington, Indiana, USA
}

Running Title: Proton Therapy for Head and Neck Adenoid Cystic Carcinoma

Funding Acknowledgements: Supported in part by the Jesse N. Jones, III Memorial Fund for Head and Neck Cancer Research at the Indiana University Melvin and Bren Simon Cancer Center.

Presented in part at the 95 $5^{\text {th }}$ annual meeting of the American Radium Society, April 27 - May 1, 2013, Scottsdale, Arizona.

Financial Disclosures and Conflicts of Interest Notification: None

Keywords: Adenoid cystic carcinoma, proton therapy, head and neck, dose-escalation, radiation therapy

Correspondence to:

Mark W. McDonald, MD

Winship Cancer Institute of Emory University

1365 Clifton Rd NE, Suite A1341

Atlanta, GA 30322

Phone: 404.778 .3473

Fax: 404.778.4139

Email: mark.mcdonald@emory.edu

This is the author's manuscript of the article published in final edited form as:

Linton, O. R., Moore, M. G., Brigance, J. S., Summerlin, D.-J., \& McDonald, M. W. (2015). Proton therapy for head and neck adenoid cystic carcinoma: Initial clinical outcomes. Head \& Neck, 37(1), 117-124.

http://doi.org/10.1002/hed.23573 


\section{ABSTRACT}

Background: To report outcomes of proton therapy in head and neck adenoid cystic carcinoma.

Methods: Retrospective analysis of 26 patients treated between 2004-2012. Twenty (77\%) had base of skull involvement; 19 (73\%) were treated for initial disease, 7 (27\%) for recurrent disease. Twenty were treated postoperatively, 6 after biopsy alone and 24 had positive margins or gross residual disease. Median dose delivered was 72 Gy (RBE).

Results: Median follow-up was 25 months (range 7-50). 2-year overall survival was 93\% for initial disease course and 57\% for recurrent disease ( $\mathrm{p}=0.19$ ). 2-year local control was $95 \%$ for initial disease and $86 \%$ for recurrent disease $(\mathrm{p}=0.48)$. 2-year distant metastatic rate was $25 \%$. Late toxicity of grade 0 or 1 was seen in 17 patients, grade 2 in 5 , grade 3 in 2 , grade 4 in 1 , and grade 5 in 1.

Conclusions: Initial outcomes of proton therapy are encouraging. Longer follow-up is required. 


\section{INTRODUCTION}

Adenoid cystic carcinoma (ACC) is an uncommon secretory gland tumor most often arising in salivary tissue in the head and neck. Its natural history is marked by a propensity for perineural tumor spread and both local recurrence and distant metastatic disease. Many patients have a history of slow indolent tumor growth with a prolonged clinical course, but those with lymphatic invasion, solid tumor histology, or high-grade transformation appear to have a more aggressive clinical course and reduced survival. ${ }^{1,2,3}$ The incidence of ACC appears to be decreasing over time, and there is suggestion that survival is superior in women compared to men. ${ }^{4}$ Surgical resection and postoperative radiotherapy are the standard of care, while systemic therapy has been of little utility. ${ }^{5}$

Patients with advanced tumor stage disease ${ }^{6,7,8}$ and involvement of the skull base have poor outcomes, ${ }^{9,10}$ as disease cannot usually be surgically extirpated and the lower radiation tolerance of closely adjacent critical normal structures such as the optic apparatus, brainstem, and temporal lobes can preclude delivery of radical doses of radiotherapy. In these patients, local disease control is of special importance, given the profound sequelae of disease progression at the skull base.

Given the suboptimal results of x-ray based radiation therapy for patients with unresectable or gross residual disease or those with skull base involvement, ${ }^{6,9,11,12,13}$ dose intensification has been explored. Prior studies have suggested a dose response relationship for ACC, ${ }^{14,15,16}$ where higher doses seem to be associated with improved and durable local tumor control. Neutron therapy has been used to provide a biologically more potent dose of radiation therapy for patients with unresectable salivary gland tumors: although the 5-year locoregional control rate for patients with ACC and skull base involvement was a disappointing $15-23 \%,{ }^{11,12}$ though improved with addition of a gamma knife radiosurgery boost. ${ }^{17}$

Proton therapy is a modality of radiation therapy with a biologic effect normalized to be identical to megavoltage Xrays, but with superior dose localization, facilitating dose-escalation while respecting the dose constraints of surrounding normal structures. ${ }^{18}$ We report initial clinical outcomes of proton therapy at our institution for head and neck ACC.

\section{MATERIALS AND METHODS}

Institutional review board approval was obtained for this retrospective review. Twenty-seven consecutive patients were treated at the Indiana University Health Proton Therapy Center between June 2004 and April 2012 for head and neck adenoid cystic cancer. One patient, who was reirradiated with palliative intent (30 Gy in 10 fractions), was excluded from analysis. The remaining 26 patients were treated with curative intent: 19 (73\%) were treated for their initial disease course 
and 7 (27\%) were treated for recurrent disease. Twenty patients (77\%) were treated after surgery, and 18 of these (90\%) had positive margins or gross residual disease. The remaining 6 patients (23\%) were treated after biopsy alone.

Skull base involvement was defined as tumor involving the infratemporal fossa, clivus, foramen ovale, foramen rotundum, cavernous sinus, sphenoid sinus, orbit, or intracranial tumor extension. In the subset of 19 patients treated in their initial disease presentation, 14 had skull base involvement and none had metastatic disease at presentation. In the subset of 7 patients treated for recurrent disease, 6 had received prior head and neck radiation, 6 had skull base involvement, and 3 had known distant metastatic pulmonary disease, but received aggressive local therapy due to the clinically indolent nature of their distant metastases and the importance of obtaining local control. Table 1 lists patient characteristics. Half the patients were treated by the senior author.

Included in the analysis are two patients who received some component of photon therapy. One patient was referred for a proton therapy boost after initial delivery of 61.2 Gy of intensity modulated photon radiation therapy (IMRT) at an outside institution. One patient received 14.4 Gy of IMRT before beginning proton therapy due to her weight initially being in excess of our table tolerance. The remaining patients were treated exclusively with proton therapy.

Proton treatment planning involved creation of custom immobilization devices including a thermoplastic face mask, alpha cradle body mold, and acquisition of a non-contrast treatment planning CT scan with 1 mm slice thickness. Preoperative and postoperative imaging was coregistered to delineate the initial extent of tumor, areas of residual disease, and postoperative anatomy. The target volume covered the primary tumor bed and course of the initial tumor and included coverage of the skull base, tracking any involved cranial nerve through the skull base foramina to the surface of the brainstem. Ipsilateral cervical lymphatics were irradiated in the 1 node positive patient. Limited regional nodal irradiation was delivered electively in 7 other patients per the preference of one of the treating physicians.

Proton treatment planning was three-dimensional conformal radiotherapy using uniform scanning beam delivery, which delivers a uniform spread out Bragg peak across each field. ${ }^{19}$ The choice of beam angles and number of beams varied by the extent and complexity of each individual patient's tumor. Brass apertures were fabricated to define the shape of each field and conform to the lateral extent of the target, with Lucite range compensators milled to shape the distal end of the proton beam, achieving distal conformality to the target. Treatment optimization involved multiple iterative adjustments in individual field shape and design to achieve the desired target coverage and normal tissue sparing. Our institutional practice is to use a $2 \mathrm{~mm}$ expansion on clinical target volumes to create a planning target volume, which accounts for patient setup uncertainties. Individual beam parameters including compensator smearing were selected with consideration of the 
surrounding tissue heterogeneities and potential uncertainties inherent in each unique beam path. Range uncertainty is dependent on depth or energy but generally 2-4 mm in the head and neck. Details of our proton beam delivery system have been previously published. ${ }^{20}$ Daily kilovoltage orthogonal X-ray images were taken to align the patient prior to treatment of each field, with adjustments made on a robotic patient positioner with six degrees of freedom to achieve near stereotactic treatment alignment, verified by a physician prior to treatment delivery. ${ }^{21}$

Patients were treated with once daily fractionation of 1.8 - 2 Gy (RBE). Proton dose is expressed in Gy (RBE) with a relative biologic effectiveness of 1.1 compared to megavoltage X-ray therapy. A strategy of high dose radiotherapy was pursued per institutional approach, based on the prior published experience with high-dose proton radiotherapy in this disease. ${ }^{22}$ The total dose administered was 75.6 Gy (RBE) for gross disease and 66 - 70.2 Gy (RBE) for negative margins. For those with positive margins, the dose was 70.2 - 72 Gy (RBE), attenuated to $66.6 \mathrm{~Gy}$ (RBE) in 1 patient due to proximity to the optic nerve in a patient with a single sighted eye, and to $68.4 \mathrm{~Gy}(\mathrm{RBE})$ in 1 patient due to physician preference. No patient received concurrent systemic therapy.

Local control (LC), distant metastasis (DM), and overall survival (OS) were the events analyzed. Local tumor recurrence was defined as enlargement of residual tumor or regrowth of tumor at the primary tumor site or within or adjacent to the volume of the preoperative tumor extent. Patients with metastatic disease at the time of treatment (n=3) were excluded from analysis in determination of the estimate of the risk of developing DM. The time of follow-up was measured from the end date of radiation treatment. Two year event estimates were calculated using the Kaplan Meier method and 95\% confidence intervals (CI) are presented. The log-rank test was used to perform univariate analysis of patient and disease characteristics. Variables with a p value $<0.2$ on univariate analysis were selected for inclusion in a Cox multivariate regression analysis using block entry method to explore association with local control and overall survival.

Information on acute and late toxicity was retrospectively gathered from weekly treatment status notes, the treatment completion summary, follow-up notes, and correspondence from follow-up with other physicians and graded according to the Common Terminology Criteria for Adverse Events (version 4.0) of the National Cancer Institute.

\section{RESULTS}

The median follow-up on all patients was 25 months (range 7 - 54 months) and 27 months in patients that are alive. The 2-year estimate of OS was 82\% (95\% CI 66-98\%). The 2- year estimate of LC was 92\% (95\% CI 82-100\%). The 2-year estimate for the development of DM (23/26 patients in analysis) was 25\% (95\% CI 5-45\%). Distant metastasis developed in 
five patients at 1 through 25 months after treatment. Four patients had DM as the initial site of relapse. One had synchronous local and distant metastases. No patients developed cervical nodal recurrence.

Univariate analysis did not demonstrate a statistically significant relationship between any patient or treatment variable and local control or overall survival. The variables analyzed were initial versus recurrent disease, presence or absence of skull base involvement, gender, age (continuous variable), primary tumor site, surgical resection versus biopsy alone, solid versus non-solid histology, positive versus negative margin status, perineural invasion, lymphovascular space invasion, presence or absence of gross residual disease, $\mathrm{T}$ stage, $\mathrm{N}$ stage, group stage, total dose (continuous variable), and prior radiation versus radiation-naïve.

Multivariate Cox regression analysis for overall survival in a model including the variables age $(\mathrm{p}=0.16)$, prior radiation $(p=0.06)$, lymphovascular space invasion $(p=0.06)$, presence of gross residual disease $(p=0.17)$, perineural invasion $(\mathrm{p}=0.17)$, and skull base involvement $(\mathrm{p}=0.17)$ yielded no variables with a statistically significant association with overall survival. Multivariate Cox regression analysis for local control in a model including the variables gender ( $\mathrm{p}=0.064)$, and age ( $\mathrm{p}=0.11)$ yielded no variables with a statistically significant association with local control.

The 2-year LC for patients treated for their initial disease course was 95\% (95\% CI 85\% - 100\%) and for recurrent disease 86\% (95\% CI 60-100\%) (Figure 1, p=0.48). The 2-year OS for those treated for initial disease was 93\% (95\% CI 81100\%) versus 57\% for those with recurrent disease (95\% CI 20-94\%) (Figure 2, p=0.19). The 2-year LC for patients without skull base involvement was 100\% and 90\% (95\% CI 77\%-100\%) for those with skull base involvement (Figure 3, p=0.21). The 2-year OS for those without skull base involvement was 100\% compared to 77\% (95\% CI 58-97\%) for those with skull base disease (Figure $4, \mathrm{p}=0.17)$.

The 2-year LC for patients irradiated after biopsy only (n=6) was $100 \%$ compared to $90 \%$ (95\% CI 77-100\%) for patients treated with surgery and postoperative radiation $(\mathrm{p}=0.97)$. The 2-year LC for patients treated with macroscopic residual disease with or without surgical resection (n=12) was 93\% (95\% CI 79-100\%) compared to 92\% (95\% CI 76-100\%) for patients treated without gross residual disease $(p=0.64)$.

Fourteen patients with skull base involvement were treated with proton therapy in their initial disease presentation. Among these patients, the median follow-up time was 27 months (range 11-50 months). The 2-year estimates of LC, OS and DM were 93\%, 91\%, and 32\% respectively (Figure 5). 
Among the 7 patients with recurrent disease, 6 had received one or more courses of prior radiation therapy. The median follow-up for these 7 patients was 24 months (range 7-43 months). The 2-year estimates of LC, OS and DM were $86 \%, 57 \%$, and $25 \%$ respectively.

\section{Pattern of Failure Analysis}

Isolated local recurrences occurred in two patients at 3 months and 45 months after treatment, respectively. Both tumors originated in the ethmoid sinus, involved the base of skull, and both patients had gross residual disease at the time of radiation. One patient had been treated for recurrent, previously irradiated disease and progressed within the 75.6 Gy (RBE) dose region. The second patient was treated for their initial disease presentation, receiving radiation only to the postoperative volume of residual tumor, and developed recurrent tumor within the primary tumor bed, which, for unclear reasons, was excluded from the radiation treatment volume. It was noted that in this patient, the preoperative MRI had not been coregistered to aid in delineation of the initial disease extent. One additional patient with a nasal cavity primary developed an orbital apex recurrence accompanied by bone metastases 2 months after radiation therapy, and was noted to have suboptimal coverage of the foramen rotundum.

\section{Treatment Toxicity}

Acute toxicity included transient radiation dermatitis of maximum grade II in 16 patients, lateralized mucositis of maximum grade II in 7 patients, and 2 patients with grade II serous otitis. Of the two patients treated for orbital tumors with an intact eye, one developed an acute grade III keratitis requiring treatment to be held temporarily for resolution. One other patient with a sinonasal primary had a brief treatment interruption for initiation of treatment of a nasal cavity methicillinresistant staphylococcus aureus infection. All patients completed the prescribed course of radiation.

Late toxicity of grade 0 or 1 was seen in 17 patients, grade 2 in 5 patients, grade 3 in 2 patients, grade 4 in 1 patient, and grade 5 in 1 patient. The grade 3 toxicities included mandibular osteoradionecrosis in one patient treated for initial disease in whom $9 \mathrm{~cm}^{3}$ of the mandible (11.6\% relative volume) received a dose of $70 \mathrm{~Gy}$ (RBE) or higher, with a maximum mandible dose of 82.9 Gy (RBE). The mandible had not been contoured by the treating physician, which may explain why alternate beam arrangements were not used to keep the mandible at acceptable dose constraints. Hyperbaric oxygen therapy was employed with improvement. A second patient who previously received 50.4 Gy of photon therapy was reirradiated for recurrent parotid disease six years later to $72 \mathrm{~Gy}$ (RBE) and 27 months later developed grade 3 otologic toxicity requiring tympanic membrane reconstruction. The grade 4 toxicity was anticipated unilateral vision loss in a patient who was irradiated for an orbital tumor that directly involved the optic nerve and consented to the expectation of vision loss. The 
single case of late grade 5 toxicity developed in a patient with a history of an extensive nasopharyngeal adenoid cystic carcinoma previously irradiated with 54 Gy of IMRT and who later received 3 Gamma Knife radiosurgery treatments to different areas of recurrent head and neck disease over a 6 year timeframe. None of the patient's prior radiation treatment plans had been archived in digital format, so it was impossible to accurately reconstruct the lifetime composite dose distribution. Two years after the most recent prior radiation, in the absence of distant metastatic disease, the patient was reirradiated to $75.6 \mathrm{~Gy}$ (RBE) to progressive painful gross recurrent disease in the infratemporal fossa and frontal sinus. The patient had radiographic and clinical regression of disease and improvement in pain, but six months later developed a cerebrospinal fluid leak and meningitis, and subsequently died.

\section{DISCUSSION}

Pommier and colleagues have reported results of high-dose proton therapy for patients with skull base ACC, finding a 5-year local control of 93\% (100\% at 2 years) in 23 patients who had received a median dose of 75.9 Gy (RBE), mostly treated with an hyperfractionated accelerated concomitant boost technique. ${ }^{22}$ These results are encouraging in a population of patients with advanced, complex disease, and support a strategy of dose escalation, which was accomplished with acceptable toxicity using proton therapy. All of these patients received some component of photon therapy (median proton component was $60 \%$ of the dose), due in part to the limited clinical availability of the former Harvard Cyclotron Laboratory, in contrast to our own experience, which used once daily fractionation and exclusively proton therapy in all but two patients. Among our subset of 14 patients with base of skull involvement treated for initial disease presentation, the 2year local control was 100\% after a median dose of 72 Gy (RBE) in once daily fractionation.

A review of the literature on ACC which reports results by base of skull involvement or T stage (Table 2) suggests that the results achieved with high-dose proton therapy are among the most favorable reported in the literature for patients with advanced stage disease. Such comparisons among different retrospective clinical series should be interpreted with caution as there are marked limitations including the inability to control for multiple prognostic factors and selection biases.

Despite these limitations, we believe these data support dose intensification for high risk ACC based on the promising initial local control. Our institutional approach to patients with adenoid cystic carcinoma is surgical resection and postoperative radiotherapy, given that multiple series have suggested that patients treated with radiotherapy alone have inferior disease control and survival compared to those treated with surgery and postoperative radiotherapy. Skull base involvement and the probability of positive margins or gross residual disease in the cavernous sinus does not in our opinion 
preclude resection of extracranial disease with the plan for postoperative high dose proton therapy. For patients with skull base involvement, gross residual disease, or positive margins, we utilize high dose postoperative proton therapy. Patients who decline surgery or are inoperable may be treated with palliative intent, referred for consideration of neutron therapy, or treated with high-dose proton therapy depending on clinician judgment of the feasibility of delivery of radical doses of radiation. Excellent results have been reported with postoperative standard dose photon therapy among patients with negative margins. ${ }^{15}$ However, in patients with earlier stage disease in more favorable sites where there is less rationale for dose escalation, proton therapy may offer an advantage in mitigating toxicities of therapy. Figure 6 illustrates a clinical example where proton therapy was used to eliminate oral cavity irradiation and preserve salivary gland function in a patient with a buccal mucosa ACC.

Patients treated more intensively, either with high-dose photon ${ }^{6}$ or proton ${ }^{22}$ therapy, or neutron therapy with radiosurgical boost, ${ }^{17}$ appear to have improved disease control compared to older series of non-operative management with lower dose photon therapy, raising the possibility of using more conformal radiotherapy techniques for non-surgical management in patients for whom radical resection would be excessively morbid. The comparatively poor results seen in series of patients treated with radiation therapy alone for advanced disease may reflect delivery of insufficient dose of radiotherapy for gross disease, often complicated by proximity of unresectable disease to critical normal structures that have a lower tolerance dose to radiotherapy. In our series, 6 patients were treated after biopsy alone, and 14 with macroscopic residual disease, with results that did not appear inferior to those treated postoperatively for microscopic residual disease. Similarly, in the series by Pommier and colleagues, local disease control was maintained at both 5 and 10 years among all 11 patients treated for skull base disease with high-dose proton therapy after biopsy only.

Our experience with high dose proton therapy reflects a short median follow-up time, and more mature follow-up is desired in this disease with a long natural history. The data for high dose radiotherapy is retrospective and has not been rigorously compared against standard dose radiotherapy, which limits the conclusions which can be drawn regarding the role of dose escalation in obtaining local control.

Late treatment toxicity in patients treated for their initial disease course was modest with 1 patient developing grade 3 toxicity which is not surprising given the large volume of mandible treated to a high dose, and 1 patient with grade 4 vision loss which was anticipated in treatment of an orbital tumor involving the optic nerve in a patient who otherwise would have required enucleation. Additional late toxicity was seen in the smaller subset of patients treated for recurrent disease, including one death due to cerebrospinal fluid leak and meningitis in a heavily pretreated patient. Given the small numbers 
involved, there was no statistically significant difference in toxicity among the treatment naïve and previously irradiated cohorts.

Our experience with 7 patients with recurrent disease, 6 of whom had previously been irradiated, demonstrated a surprisingly good 2-year LC of 86\%. In this subset of patients with particularly challenging disease complicated by one or more prior courses of radiation therapy, high-dose proton therapy was pursued in an effort to obtain control of skull base disease causing or threatening significant clinical deficits. In one patient who had undergone prior IMRT and 3 radiosurgery procedures, reirradiation may have contributed to subsequent cerebrospinal fluid leak, meningitis, and death, which underscores the potential for significant toxicity with reirradiation. The safety and efficacy of reirradiation in this complex cohort of patients cannot be evaluated from this small subset. Comprehensive reviews of the role of reirradiation in head and neck cancer have been published. ${ }^{23}$

Additional limitations of this study include its retrospective nature, the relatively small number of patients, and the short median follow-up time, with longer follow-up necessary to assess the durability of local disease control. Our study benefits from a relatively uniform treatment approach to these patients over the study period. The importance of careful treatment planning and the complexity of care involved in treatment of patients with skull base disease is underscored by the pattern of failure analysis which revealed that $2 / 3$ of the local failures may have been prevented by changes in treatment volume.

While optimizing local control and reducing toxicity of local therapy remains an important goal of future research, the insidious course of adenoid cystic carcinoma and the high propensity for distant metastatic disease ${ }^{24}$ underscore the importance of continued efforts in evaluating the role of systemic therapies and developing novel agents with activity against ACC.

\section{CONCLUSIONS}

In our initial clinical experience among patients with mostly advanced stage head and neck adenoid cystic carcinoma, high-dose proton therapy provides encouraging preliminary local control. Longer follow-up is needed to gauge the durability of disease control and to monitor for late toxicities of therapy. Careful treatment planning is essential to optimize outcomes. 


\section{Table 1: Patient Characteristics}

\section{Characteristic}

Number (\%)

Gender

Male

$12(46 \%)$

Female

$14(54 \%)$

Age (years)

Median

46

Range

21-73

Histology

Cribriform/Tubular

$18(69 \%)$

Solid

$8(31 \%)$

Primary Tumor Site

Ethmoid Sinus

$2(8 \%)$

Maxillary Sinus

$5(19 \%)$

Nasal Cavity

$2(8 \%)$

Nasopharynx

$2(8 \%)$

Orbit

$4(15 \%)$

Parotid Gland

$7(26 \%)$

Submandibular Gland

$4(15 \%)$

Major Salivary

$11(42 \%)$

Minor, Accessory, or Ectopic Salivary

$15(58 \%)$

Skull Base Involvement

Cavernous sinus

$9(35 \%)$

Foramen rotundum

$6(23 \%)$

Foramen ovale

$6(23 \%)$

Infratemporal fossa

$6(23 \%)$

Orbit

$4(15 \%)$ 
Clivus

Sphenoid Sinus

Treated at time of

Initial Presentation

19 (73\%)

Recurrent Disease

7 (27\%)

Base of Skull

Involved

Not Involved

$6(23 \%)$

Margin Status

Positive

Negative

$2(8 \%)$

Gross Residual Disease Present

Yes

$12(46 \%)$

No

14 (54\%)

Perineural Invasion

Yes

$19(73 \%)$

No

7 (27\%)

Lymphovascular Space Invasion

Yes

8 (31\%)

No

$18(69 \%)$

Bone Invasion

Yes

$14(54 \%)$

No

12 (46\%)

T Stage

II

0

III

$4(15 \%)$

IV

$20(77 \%)$ 
N Stage

N0

25 (96\%)

N2

$1(4 \%)$

M Stage

M0

M1

Surgery Prior to Proton Therapy

Yes

$20(77 \%)$

No

$6(33 \%)$

Neck Dissection

Yes

No

$13(50 \%)$

Type of Surgery

Parotidectomy

with facial nerve preservation and infratemporal fossa resection

with facial nerve sacrifice, infratemporal fossa resection

with facial nerve sacrifice, infratemporal fossa resection, segmental mandibulectomy

with facial nerve preservation

Orbital Exenteration

Orbital Exenteration, Anterior skull base resection, maxillectomy

Orbital Debulking

Maxillectomy with orbital floor resection 1

Maxillectomy with orbital floor resection, infratemporal fossa resection $\quad 1$

Transnasal, transethmoid anterior skull base resection $\quad 1$

$\begin{array}{ll}\text { Transoral, transpalatal partial nasopharyngectomy } & 1\end{array}$

Nd:YAG* laser transmaxillary debulking of maxillary and nasopharyngeal tumor 1

Total rhinectomy with transnasal, transethmoid anterior skull base resection $\quad 1$

Wide local excision of buccal mucosal tumor $\quad 1$ 
Submandibulectomy

Submandibulectomy with partial mandibulectomy

\section{Extent of Surgery}

Gross Total Resection

$14(54 \%)$

Subtotal Resection

$8(31 \%)$

Biopsy Only

$4(15 \%)$

Prior Radiation Therapy

Yes

$6(23 \%)$

No

$20(77 \%)$

Proton Dose in Gy (RBE)

Median

72

Range

$66-75.6$

* Nd:YAG $=$ neodymium-doped yttrium aluminum garnet 
Table 2: Recent series of local control and overall survival for high risk head-and-neck adenoid cystic

carcinoma

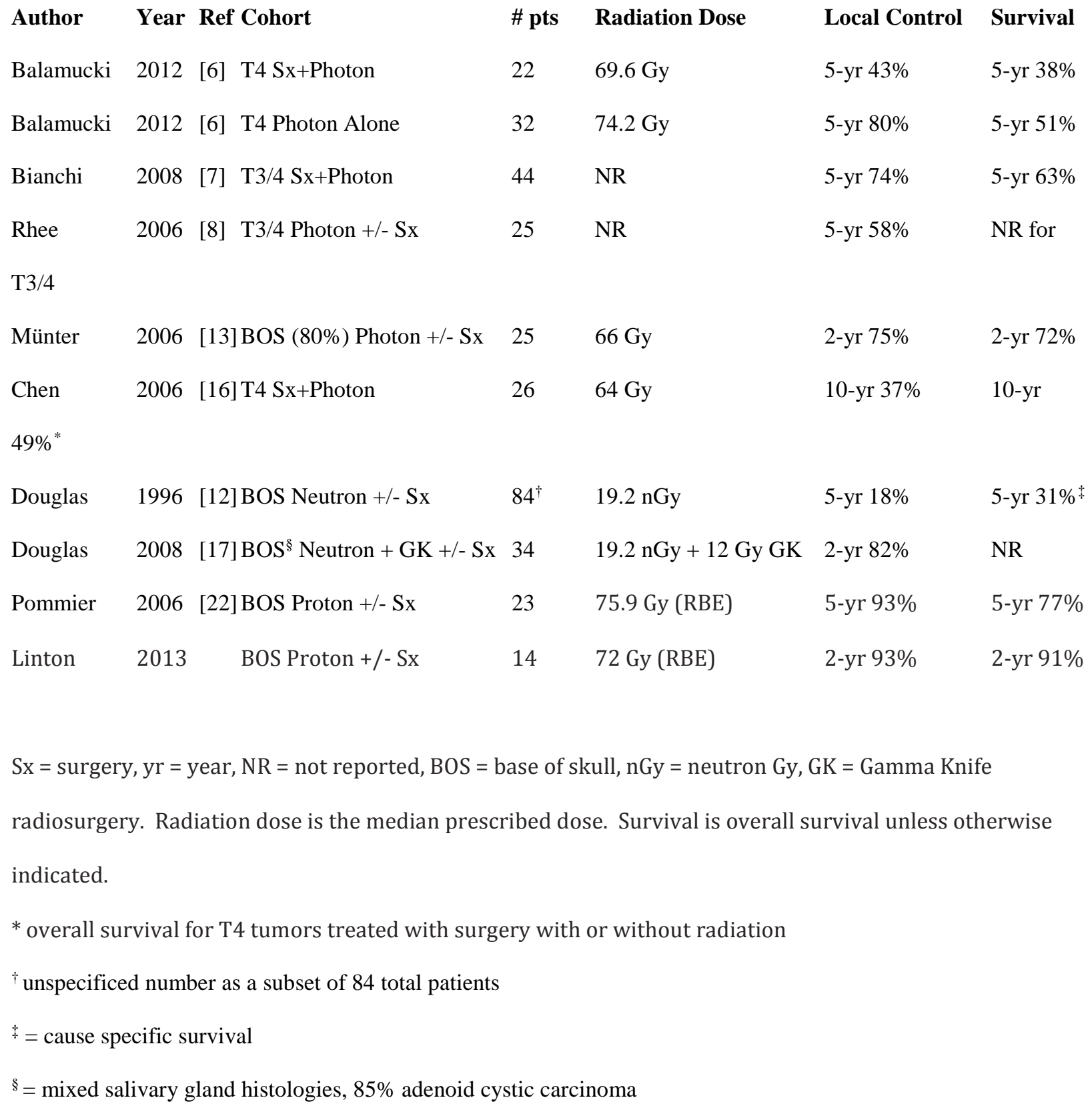


Figure 1: Local control for patients treated for initial versus recurrent disease.

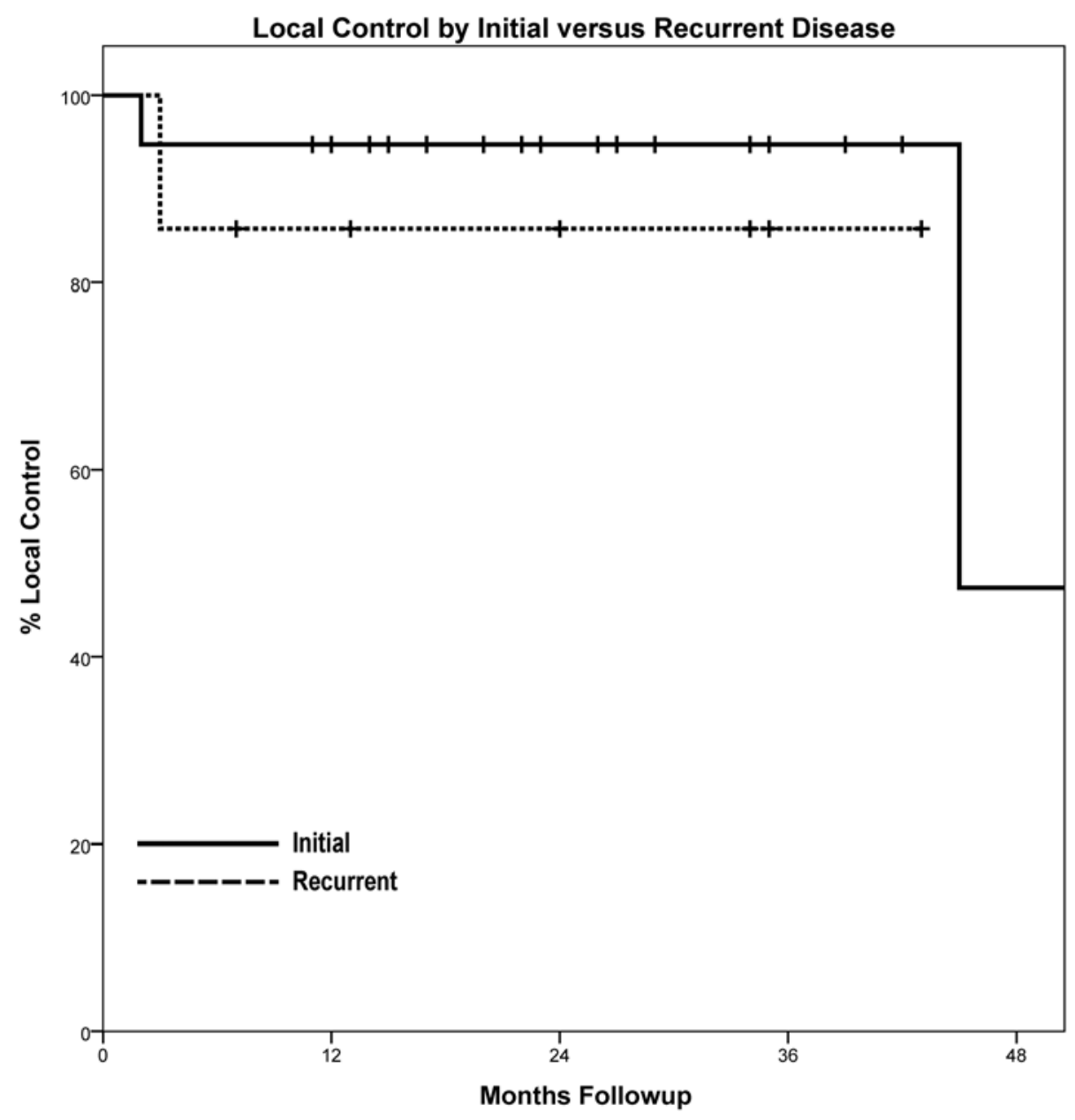


Figure 2: Overall survival for patients treated for initial versus recurrent disease.

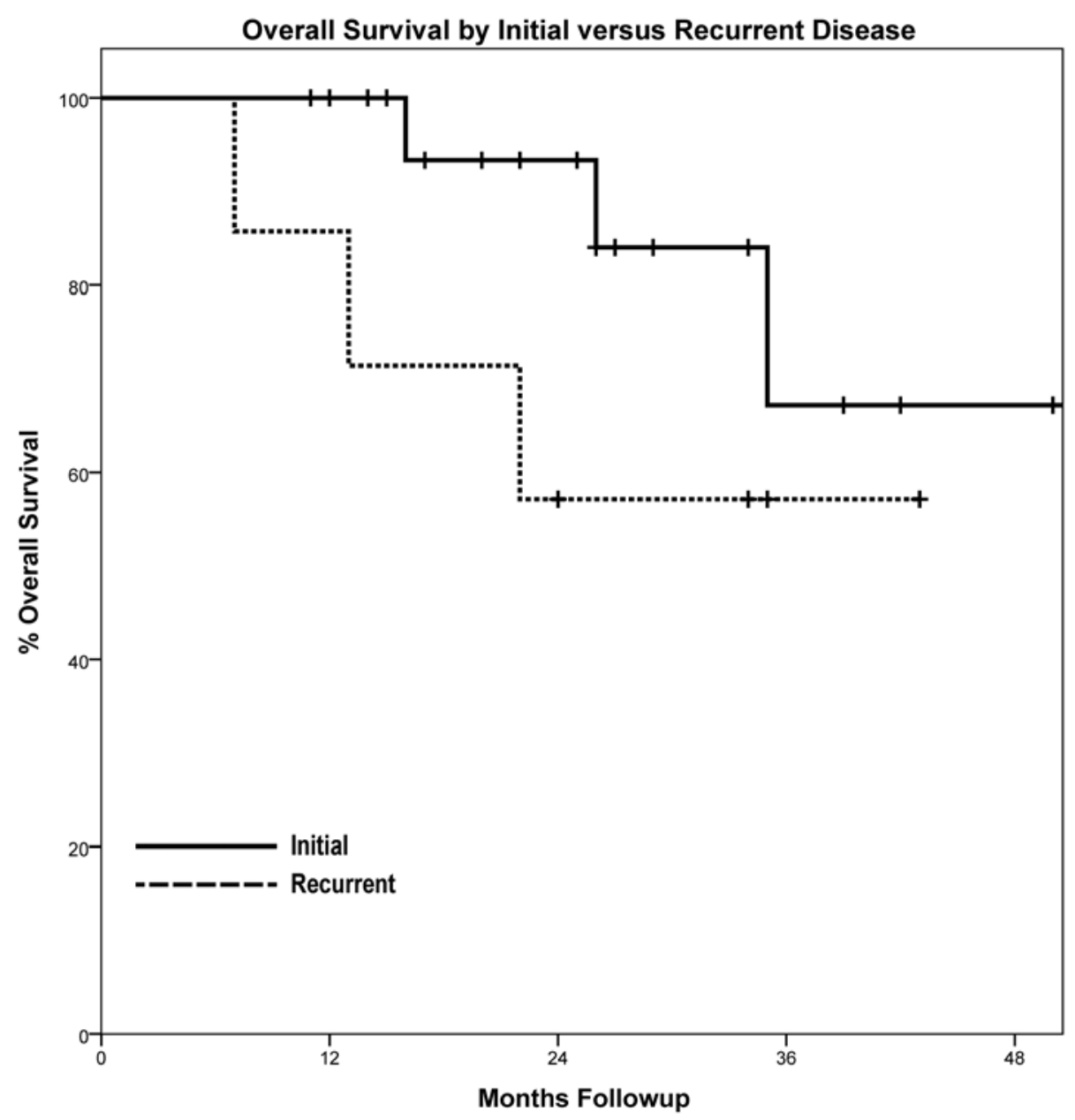


Figure 3: Local control for patients treated for skull base versus non-skull base disease.

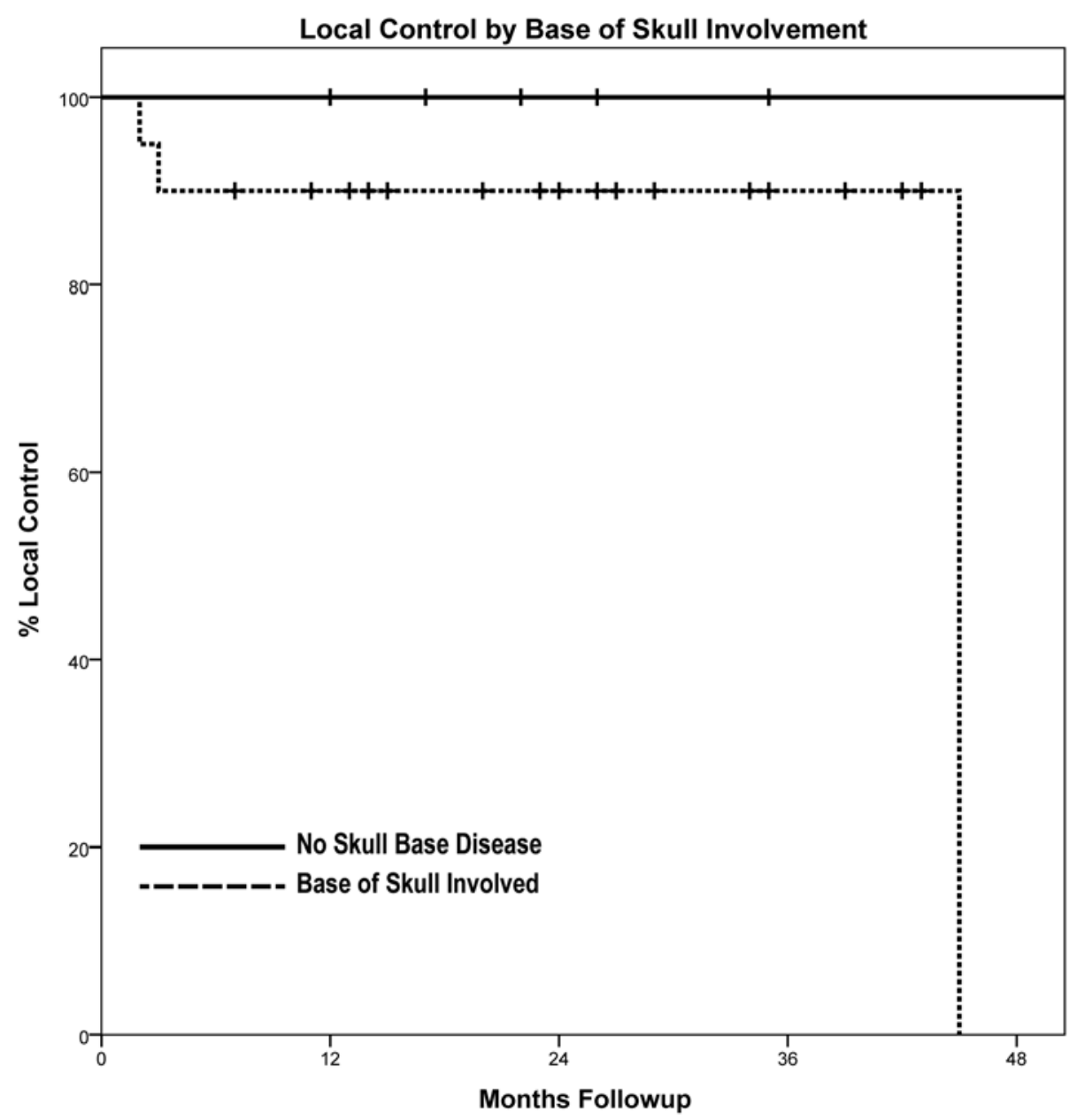


Figure 4: Overall survival for patients treated for skull base versus non-skull base disease.

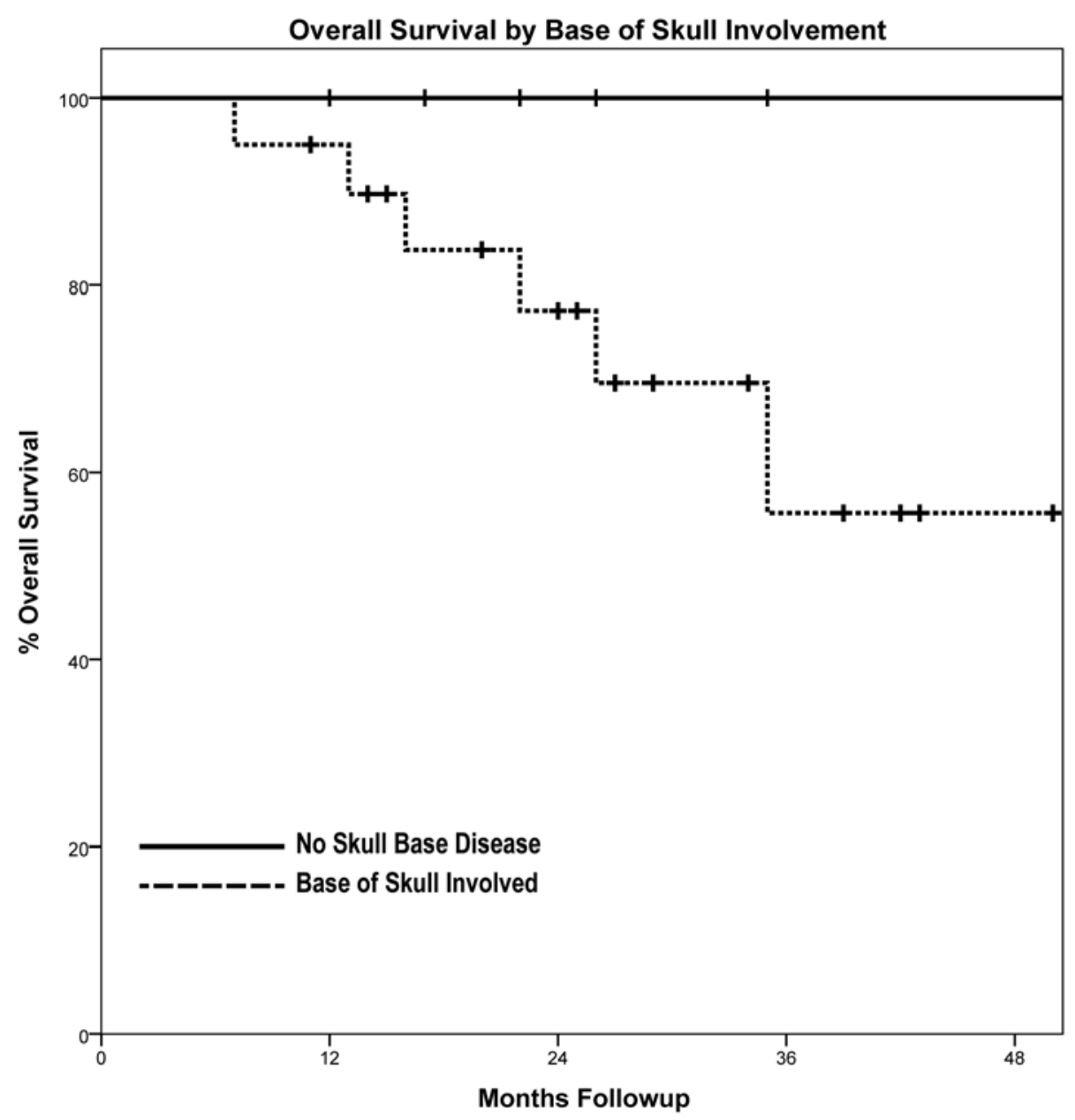


Figure 5: Local control, overall survival, and distant metastasis in patients treated for initial disease with skull base involvement.

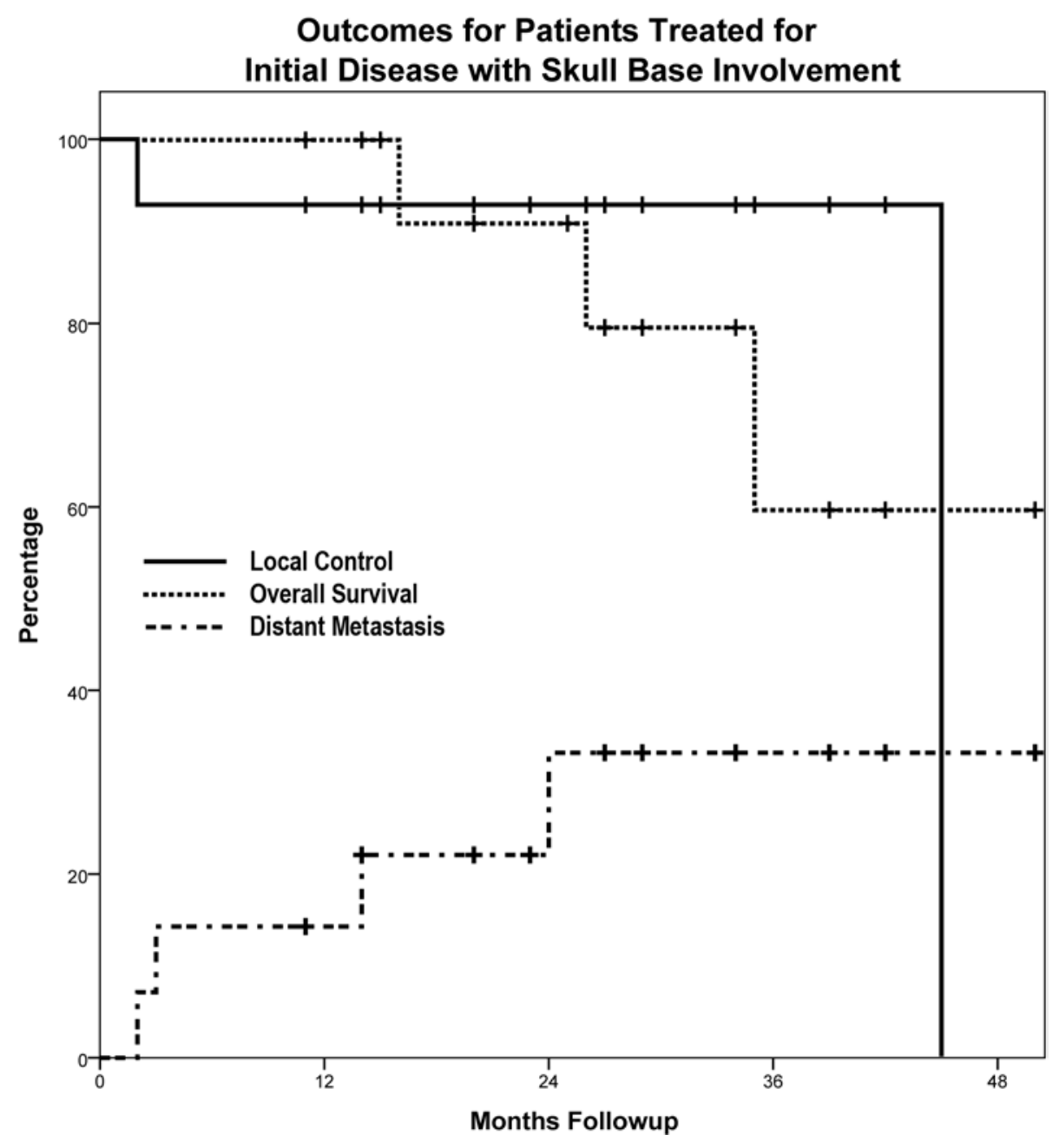


Figure 6: Example of proton therapy for early stage oral cavity adenoid cystic carcinoma.
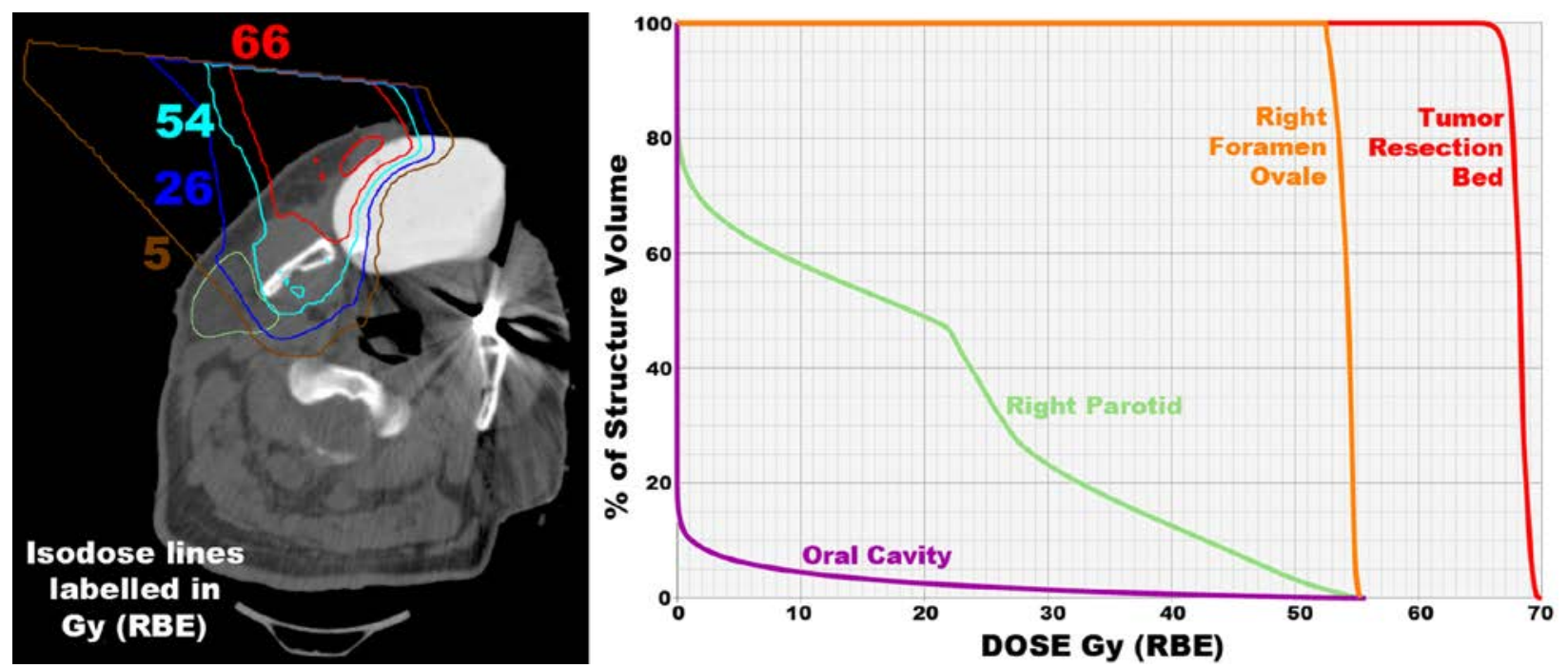

This patient underwent excision of a $1.2 \mathrm{~cm}$ adenoid cystic carcinoma from the right buccal mucosa with negative but close surgical margins, stage I, pT1 N0 M0. Perineural invasion was present on pathology. Proton therapy was used to treat the tumor bed to a dose of 66 Gy (RBE) in 33 fractions. A custom mouth guard was fabricated to completely shield the gingiva and oral cavity, allowing the distal end of the proton beam to terminate in the mouth guard rather than in normal tissues. The initial treatment volume included elective irradiation along the course of the mandibular nerve to foramen ovale to $54 \mathrm{~Gy}$ (RBE), with proton beam geometry selected to spare the ipsilateral parotid. The patient developed grade 2 mucositis confined to the treated portion of the ipsilateral buccal mucosa but developed no taste perversion, has no xerostomia and has experienced no dental decay 17 months after therapy. On the left is an axial slice from the treatment planning CT with color isodose lines. The tumor bed is encircled in red and the ipsilateral parotid gland in green. On the right is the treatment plan dose volume histogram showing the sparing of the oral cavity and ipsilateral parotid gland. 


\section{REFERENCES}

${ }^{1}$ Bhayani MK, Yener M, El-Naggar A, et al. Prognosis and risk factors for early-stage adenoid cystic carcinoma of the major salivary glands. Cancer 2012;118:2872-2878.

${ }^{2}$ da Cruz Perez DE, de Abreu Alves F, Nobuko Nishimoto I. Prognostic factors in head and neck adenoid cystic carcinoma. Oral Oncol 2006;42:139-146.

${ }^{3}$ Seethala RR, Hunt JL, Baloch ZW, et al. Adenoid cystic carcinoma with high-grade transformation: a report of 11 cases and a review of the literature. Am J Surg Pathol 2007;31:1683-1694.

${ }^{4}$ Ellington CL, Goodman M, Kono SA, et al. Adenoid cystic carcinoma of the head and neck: incidence and survival trends based on 1973-2007 Surveillance, Epidemiology, and End Results data. Cancer 2012;118:44444451.

${ }^{5}$ Papaspyrou G, Hoch S, Rinaldo A, et al. Chemotherapy and targeted therapy in adenoid cystic carcinoma of the head and neck: a review. Head Neck 2011;33:905-911.

${ }^{6}$ Balamucki CJ, Amdur RJ, Werning JW, et al. Adenoid cystic carcinoma of the head and neck. Am J Otolaryngol 2012;33:510-518.

${ }^{7}$ Bianchi B, Copelli C, Cocchi R, et al. Adenoid cystic carcinoma of intraoral minor salivary glands. Oral Oncol 2008;44:1026-1031.

${ }^{8}$ Rhee C, Won T, Lee CH, et al. Adenoid cystic carcinoma of the sinonasal tract: treatment results. Laryngoscope 2006;116:982-986.

${ }^{9}$ Prokopakis EP., Snyderman CH., Hanna EY., et al., Risk Factors for Local Recurrence of Adenoid Cystic Carcinoma: The Role of Postoperative Radiation Therapy. 1999; 20:281-286

${ }^{10}$ Lupinetti AD, Roberts DB, Williams MD, et al. Sinonasal adenoid cystic carcinoma: The M.D. Anderson Cancer Center experience. Cancer 2007;110:2726-2731.

${ }^{11}$ Douglas JG, Laramore GE, Austin-Seymour M, et al. Treatment of locally advanced adenoid cystic carcinoma of the head and neck with neutron radiotherapy. Int J Radiat Oncol Biol Phys, 2000;46:551-557.

12 Douglas JG, Laramore GE, Austin-Seymour M, et al. Neutron radiotherapy for adenoid cystic carcinoma of minor salivary glands. Int J Radiat Oncol Biol Phys 1996;36:87-93. 
${ }^{13}$ Münter MW, Schulz-Ertner D, Hof H, et al. Inverse planned stereotactic intensity modulated radiotherapy (IMRT) in the treatment of incompletely and completely resected adenoid cystic carcinomas of the head and neck: initial clinical results and toxicity of treatment. Radiat Oncol 2006;1:17.

${ }^{14}$ Simpson JR, Thawley SE, Matsuba HM. Adenoid cystic salivary gland carcinoma: treatment with irradiation and surgery. Radiology 1984;151:509-512.

${ }^{15}$ Garden AS, Weber RS, Morrison WH, et al. The influence of positive margins and nerve invasion in adenoid cystic carcinoma of the head and neck treated with surgery and radiation. Int J Radiat Oncol Biol Phys 1995;32:619626.

${ }^{16}$ Chen AM, Bucci MK, Weinberg V, et al. Adenoid cystic carcinoma of the head and neck treated by surgery with or without postoperative radiation therapy: prognostic features of recurrence. Int J Radiat Oncol Biol Phys 2006;66:152-159.

${ }^{17}$ Douglas JG, Goodkin R, Laramore GE. Gamma Knife steretoactic radiosurgery for salivary gland neoplasms with base of skull invasion following neutron radiotherapy. Head Neck 2008;30:492-496.

${ }^{18}$ McDonald MW, Fitzek MM. Proton therapy. Curr Probl Cancer 2010 Jul-Aug;34:257-296.

${ }^{19}$ Anferov VA. Scan pattern optimization for uniform proton beam scanning. Med Phys 2009; 36:3560-3567.

${ }^{20}$ Farr JB, Mascia AE, Hsi WC, et al. Clinical characterization of a proton beam continuous uniform scanning system with dose layer stacking. Med Phys 2008;35:4945-4954.

${ }^{21}$ Allgower CE, Schreuder AN, Farr JB, et al. Experiences with an application of industrial robotics for accurate patient positioning in proton radiotherapy. Int J Med Robotics Comput Assist Surg 2007;3:72-81.

${ }^{22}$ Pommier P, Liebsch NJ, Deschler DG, et al., Proton beam radiation therapy for skull base adenoid cystic carcinoma. Arch Otolaryngol Head Neck Surg 2006;132:1242-1249.

${ }^{23}$ McDonald MW, Lawson J, Garg MK, et al. ACR appropriateness criteria: retreatment of recurrent head and neck cancer after prior definitive radiation. Expert panel on radiation oncology - head and neck cancer. Int J Radiat Oncol Biol Phys 2011;80:1292-1298.

${ }^{24}$ Al-Mamgani A, van Rooij P, Sewnaik A, Tans L, Hardillo JAU. Adenoid cystic carcinoma of parotid gland treated with surgery and radiotherapy: long-term outcomes, QOL assessment and review of the literature. Oral Oncol 2011;48:278-283. 